\title{
EARLY DETECTION OF REJECTION IN CARDIAC MRI: A SPECTRAL GRAPH APPROACH
}

\author{
Hsun-Hsien Chang and José M. F. Moura \\ Electrical and Computer Engineering \\ Carnegie Mellon University \\ \{hsunhsc,moura $\} @$ ece.cmu.edu
}

Yijen L. Wu and Chien Ho

\author{
Pittsburgh NMR Center for Biomedical Research \\ Carnegie Mellon University
}

\begin{abstract}
This paper develops an algorithm to detect abnormalities of small animals' transplanted hearts in MRI, at early stage of rejection when the hearts do not display prominent abnormal features. Existing detection methods require experts to manually identify these abnormal regions. This task is time consuming, and the detection criteria are operator dependent. We present a semi-automatic approach that needs experts to label only a small portion of the motion maps. Our algorithm begins with representing the left ventricular motions by a weighted graph that approximates the manifold where these motions lie. We compute the eigendecomposition of the Laplacian of the graph and use these as basis functions to represent the classifier. The experimental results with synthetic data and real cardiac MRI data demonstrate the application of our classifier to early detection of heart rejection.
\end{abstract}

\section{INTRODUCTION}

The current gold standard for diagnosing rejection after heart transplantation is biopsy, which is invasive and prone to sampling errors. Cardiac magnetic resonance imaging (MRI) is a non-invasive alternative to monitor rejection of an in vivo heart. Using MRI, we can observe that, in early stages, heart rejection starts from a small region, which then spreads, in late stages, to the entire myocardium [1]. Early diagnosis and treatment of rejection increases the survival rate. However, early stage rejection does not present prominent abnormal motions. To detect subtle abnormalities, cardiologists have to carefully and manually label the images. This is labor-intensive and the results vary from expert to expert. To achieve consistent classification, we need a quantitative algorithm that reduces human involvement.

We present in this paper a semi-supervised classification algorithm. The classifier is initially trained with a small

This work was supported by NIH grants, R01EB/AI-00318 and P41EB001977, to the Pittsburgh NMR Center for Biomedical Research, Carnegie Mellon University. number of normal and abnormal motions labeled by an expert, and then it classifies the remaining unlabeled motions. The classifier is developed using the framework of spectral graph theory $[2,3,4]$. We first generate motion maps of the heart through the cardiac cycle. The motion vectors are treated as samples of a Riemannian manifold embedded in the original data space. A graph representation of the data is an approximation to the manifold. We represent the heart motion map as a graph where similar motions are connected by an edge. Spectral analysis of the graph Laplacian provides a basis of functions on the graph. We use this basis to find the optimal classifier.

This paper is organized as follows. Section 2 develops in detail our classification algorithm. Section 3 tests the algorithm with synthetic data and real cardiac MRI data, demonstrating the good performance of our classifier. Finally, Section 4 concludes this paper.

\section{METHODOLOGY}

We process a sequence of cardiac MR images sampling the cardiac cycle and determine for each phase a dense motion map; i.e., at each phase $n$ in the cardiac cycle, every myocardial pixel $i$ is assigned a motion vector $\mathbf{u}_{i}[n]$. We focus in this paper on a single MRI heart slice and in a particular phase of the cardiac cycle, so we consider $\mathbf{u}_{i} \in \mathbb{R}^{2}$ and drop the time dependence $n$. The classification problem is to design a classifier $h$ such that the class label $c_{i}$ of pixel $i$ is

$$
c_{i}=c\left(h\left(\mathbf{u}_{i}\right)\right)=\left\{\begin{aligned}
1, & \text { if } \mathbf{u}_{i} \text { is normal } \\
-1, & \text { if } \mathbf{u}_{i} \text { is abnormal }
\end{aligned}\right.
$$

Or equivalently,

$$
c_{i}=\left\{\begin{aligned}
1, & \text { if } h\left(\mathbf{u}_{i}\right) \geq \tau_{c} \\
-1, & \text { if } h\left(\mathbf{u}_{i}\right)<\tau_{c}
\end{aligned}\right.
$$

where $\tau_{c}$ is a threshold.

The 2D motion vectors of the myocardium are data points in $\mathbb{R}^{2}$. We can think of these points assembling a Riemannian manifold $\mathcal{M} \subseteq \mathbb{R}^{2}$. In our case, the manifold $\mathcal{M}$ could 
be a plane, a contour, or a point. Associated with $\mathcal{M}$ is the Laplace-Beltrami operator $\Delta$ that acts on differentiable functions on $\mathcal{M}$, see [2]. When $\mathcal{M}$ is compact, $\Delta$ has a discrete spectrum and its eigenfunctions $\left\{e_{r}\right\}$, also called harmonic functions, provide an orthogonal basis for the Hilbert space of square integrable functions on $\mathcal{M}$. Since the classifier $h$ is square integrable on $\mathcal{M}$, it can be represented in terms of the harmonic functions; namely,

$$
\forall x \in \mathcal{M}: \quad h(x)=\sum_{r=1}^{\infty} a_{r} e_{r}(x) .
$$

Hence, our task becomes the problem of finding a function $h$ on $\mathcal{M}$ such that $c(h(x)) \rightarrow\{-1,1\}$, where 1 indicates normal and -1 abnormal.

We have to approximate the manifold $\mathcal{M}$ because the number of available data points is finite. Belkin and Niyogi, $[3,4]$, suggest using a graph model to represent the data; that is, the graph approximates the manifold $\mathcal{M}$ where the data lies. The Laplace-Beltrami operator is accordingly the discrete Laplacian, see [2]. In Section 2.1, we describe the graph model for our data. Similar to (3), the spectral analysis of the graph Laplacian $\mathbf{L}$ provides the basis function for the classifier, see [4]. Once we have a representation for the classifier, we use the labeled data to find the coefficients $a_{r}$, and develop a rule for classifying abnormalities in the unlabeled data. Section 2.2 details the classifier design.

\subsection{Graph Representation}

Assume that a slice of the myocardium is imaged by $N$ pixels. From two consecutive phases, we estimate a motion map $U$ that collects all motion vectors $\mathbf{u}_{i}$ :

$$
U=\left\{\mathbf{u}_{1}, \mathbf{u}_{2}, \cdots, \mathbf{u}_{q}, \cdots, \mathbf{u}_{N}\right\} .
$$

We assume that the first $q$ motion vectors in $U$ have been labeled by human experts. In the framework of spectral graph theory, we represent the set $U$ of motion vectors by a graph $G$. In $G$, vertices $v_{i}, v_{j}$ corresponding to motions $\mathbf{u}_{i}, \mathbf{u}_{j}$ are connected by an edge if the distance $\kappa_{i j}$ between $\mathbf{u}_{i}$ and $\mathbf{u}_{j}$ is smaller than a threshold $\tau_{\kappa}$. For simplicity, here, we adopt the distance measure $\kappa_{i j}$ between two vectors $\mathbf{u}_{i}$ and $\mathbf{u}_{j}$ to be the Euclidean distance, see Fig. 1,

$$
\kappa_{i j}=\left\|\mathbf{u}_{i}-\mathbf{u}_{j}\right\| \text {. }
$$

With reference to Fig. $1, \kappa_{i j}$ is the distance between $\mathbf{u}_{i}$ and $\mathbf{u}_{j}$, and $\tau_{\kappa}$ defines the neighborhood.

A graph can be represented by an adjacency matrix, whose entry $(i, j)$ is one if the vertices $v_{i}, v_{j}$ are connected, and it is zero otherwise. To take account for the different values of $\kappa_{i j}$, we use a weighted graph and a weight matrix $\mathbf{W}$. The entries of the matrix $\mathbf{W}$ in this paper are taken to be

$$
W_{i j}=\left\{\begin{aligned}
e^{-\kappa_{i j},} & \text { if } \kappa_{i j} \leq \tau_{\kappa} \\
0, & \text { if } \kappa_{i j}>\tau_{\kappa}
\end{aligned}\right.
$$

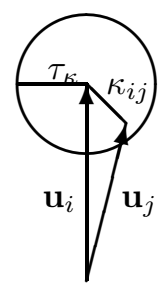

Fig. 1. Distance measure $\kappa_{i j}$ between vectors $\mathbf{u}_{i}$ and $\mathbf{u}_{j}$.

The Laplacian $\mathbf{L}$ for the graph $G$ is defined as, see [2, 4],

$$
\mathbf{L}=\mathbf{D}-\mathbf{W}
$$

where $\mathrm{D}$ is a diagonal matrix with the $i$ th diagonal entry $D_{i i}=\sum_{j} W_{j i}$. The Laplacian is a symmetric, positive semidefinite operator acting on functions defined on the vertices of the graph. Then, we solve the eigenfunction problem:

$$
\mathbf{L e}=\lambda \mathbf{e},
$$

where $\mathbf{e}$ is an eigenfunction and $\lambda$ is the corresponding eigenvalue. We index the eigenfunctions according to their eigenvalues in ascending order, $0 \leq \lambda_{1} \leq \lambda_{2} \leq \cdots \leq \lambda_{N}$. The eigenfunctions with small eigenvalues are low frequency harmonics on the graph $G$.

In order to approximate functions on the graph $G$, we pick the first $p$ eigenfunctions and form them into the eigenmatrix $\mathbf{E}$ :

$$
\mathbf{E}=\left[\mathbf{e}_{1}, \mathbf{e}_{2}, \cdots, \mathbf{e}_{p}\right] .
$$

Hence a function $\mathbf{h}$ on $G$ can be written as

$$
\mathbf{h}=\sum_{r=1}^{p} a_{r} \mathbf{e}_{r}=\mathbf{E a} .
$$

The meaning of Eq. (10) is clear. The $i$ th entry $h_{i}$ of $\mathbf{h}$ is a function mapping the motion vector $\mathbf{u}_{i}$ to a real number; namely, $h_{i}: \mathbf{u}_{i} \rightarrow \mathbb{R}$.

\subsection{Classifier}

Our task of designing a classifier reduces to finding a function $\mathbf{h}$ in (10) such that the class $c_{i}$ to which the motion $\mathbf{u}_{i}$ belongs is determined by the following rule:

$$
c_{i}=\left\{\begin{aligned}
1, & \text { if } h_{i} \geq \tau_{c}, \\
-1, & \text { if } h_{i}<\tau_{c}
\end{aligned}\right.
$$

We use the first $q$ labeled motions in $U$ to find the optimal $\mathbf{a}$ in (10). Let $\mathbf{c}$ be the $q$-dimensional vector denoting the classes of these data points; i.e., the $i$ th entry of $\mathbf{c}$ is

$$
\forall i \leq q: \quad c_{i}=\left\{\begin{aligned}
1, & \mathbf{u}_{i} \text { is labeled normal } \\
-1, & \mathbf{u}_{i} \text { is labeled abnormal }
\end{aligned}\right.
$$


Since abnormal motions are not easily distinguishable, we introduce a parameter $\alpha_{i}$ to indicate the confidence level of the experts who classify the motion $\mathbf{u}_{i}$. We let the values of the confidence level range from 0 to 1 . The higher the value, the more confident the experts. We modify the labeled class $\mathbf{c}$ to be $\overline{\mathbf{c}}$ with

$$
\bar{c}_{i}=c_{i} \alpha_{i}
$$

Vector $\overline{\mathbf{c}}$ trains the classifier by finding out the coefficients $\mathbf{a}$ in Eq. (10). The optimal a minimizes the quadratic error

$$
\varepsilon=\left\|\overline{\mathbf{c}}-\mathbf{E}_{\mathrm{lab}} \mathbf{a}\right\|^{2},
$$

where $\mathbf{E}_{\text {lab }}$ is the eigenmatrix corresponding to the $q$ labeled data points obtained from the first $q$ rows of E. Minimizing $\varepsilon$ gives $\mathbf{a}_{\mathrm{opt}}$ :

$$
\mathbf{a}_{\mathrm{opt}}=\left(\mathbf{E}_{\mathrm{lab}}^{T} \mathbf{E}_{\mathrm{lab}}\right)^{-1} \mathbf{E}_{\text {lab }}^{T} \overline{\mathbf{c}} .
$$

The remaining unlabeled motion data is classified according to the rule in Eq. (11) for all $i>q$.

\section{EXPERIMENTS}

In this section, we present experimental results. We use synthetic data to demonstrate the feasibility of the algorithm and study its performance. We then apply our algorithm to real MRI sequence of a transplanted rat heart. To run the classifier, we have to adjust two parameters: the number $p$ of eigenfunctions in Eq. (9) and the decision threshold $\tau_{c}$ in Eq. (11).

Synthetic Data: We simulate the heart as a circular hollow disk and synthesize a map of 612 motion vectors, shown in Fig. 2(a). Each pixel has a unit motion vector in the circumferential direction. We generate three regions of abnormalities marked by rectangles in Fig. 2(a). In rectangle 1, we halve the length of the vectors. In rectangle 2 , we perturb the angles of the vectors by adding to them Gaussian noise with zero mean and unit variance. In rectangle 3 , we increase the length of the vectors by the factor 1.2. An expert labels a few pixels in the normal and the two abnormal regions, inside the ellipses in Fig. 2(a). The labeled regions A, B, C with confidence levels $\alpha=1,0.5,0.75$, respectively, see Eq. (13), are used to train the classifier. We use the first 42 eigenfunctions to build the classifier and set the decision threshold $\tau_{c}=-0.4$. Fig. 2(b) shows the classification results, where the classified abnormal motions are marked by dots. The motion vectors in the three rectangle boxes are correctly classified. Abnormal regions 1 and 2 that were partially identified by the expert are correctly classified. The detector can also recognize part of the third abnormal region.

We study the performance by plotting the receiver operating characteristic (ROC). The ROC curve is a plot of the probability of hit $P_{\mathrm{H}}$ versus the probability of false alarm $P_{\mathrm{F}}$, see [5]. In this paper, we define $P_{\mathrm{H}}$ and $P_{\mathrm{F}}$ as:

$$
\begin{aligned}
& P_{\mathrm{H}}=\frac{\text { number of correctly classified abnormal pixels }}{\text { total number of abnormal pixels }} \\
& P_{\mathrm{F}}=\frac{\text { number of misclassified abnormal pixels }}{\text { total number of normal pixels }}
\end{aligned}
$$

For a given $\tau_{c}$, we get a pair of $P_{\mathrm{H}}$ and $P_{\mathrm{F}}$. We obtain the ROC curve in Fig. 2(c) by changing sequentially the threshold from -1 to 1 in increments of 0.02 ; i.e., $\tau_{c} \in$ $\{-1,-0.98, \cdots, 1\}$. This plot shows that the best operating point of the curve is at $P_{\mathrm{H}}=0.75$ and $P_{\mathrm{F}}=0.07$.

Cardiac MRI Data: Transplanted rats were studied by using heterotopic working hearts, adopting DA to BN transplant pairs. The transplanted hearts receive proper pressure pre-load and exhibit similar cardiac outputs and ventricular pressure, close to those in native hearts. We use ECG and respiration gated cine MRI to obtain images with resolution of $156 \mu \mathrm{m}$. We adopt a modified DANTE sequence for MR tagging. We cover the heart with 8 transversal slices at 10 time phases through the cardiac cycle. All MRI scans were performed on a Bruker AVANCE DRX 4.7-T system. Each image has $256 \times 256$ pixels. We use MATLAB ${ }^{\circledR}$ to implement all the algorithms.

Figure 3(a) shows a rejected rat heart imaged on the third day after transplantation. In this image, there are 2170 pixels in the myocardium. During the preprocessing step, we apply segmentation, tag line detection, and motion estimation using the algorithms developed in [6]. Fig. 3(b) shows the heart's dense motion map. In this figure, the pixels in the ellipses are labeled with the confidence level $\alpha_{\mathrm{el}}=0.8$ and the pixels in the square with $\alpha_{\mathrm{sq}}=1.0$. Two ellipses and one square indicate the labeled abnormal and normal regions, respectively, with about 80 pixels in total. We choose 112 out of possible 2170 eigenfunctions to build the classifier. The detector then automatically classifies the remaining unlabeled regions. Fig. 4 shows the detected abnormal motions marked by dots. We repeat the experiment by changing the confidence levels $\alpha_{\mathrm{el}}, \alpha_{\mathrm{sq}} \in[0.1,1]$. The classification results are not sensitive to $\alpha_{\mathrm{el}} \in[0.5,1]$ or $\alpha_{\mathrm{sq}} \in[0.7,1]$.

To evaluate the performance of the algorithm, we use contrast MRI to provide ground truth. We inject the rat with a contrast agent that binds with abnormal myocardial cells and displays dark intensities under standard cine MR imaging. Injecting a contrast agent is invasive and not desirable from a clinical point of view because it may be harmful or induce allergies.

We segment the dark abnormal regions of contrast MR images. These are the contours shown in Fig. 4. These contours provide ground truth. The ground truth illustrates that the heart has begun experiencing rejection in the epicardium. Although there are small regions of false alarms 


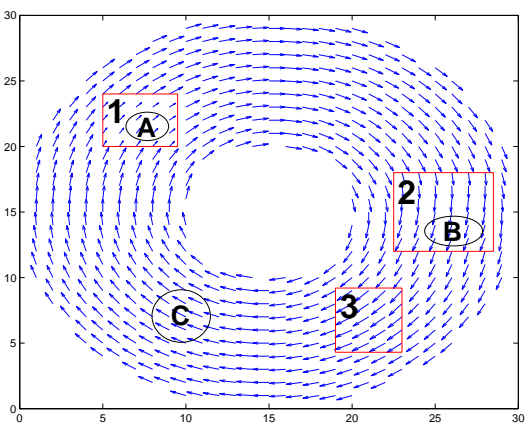

(a) Motion map.

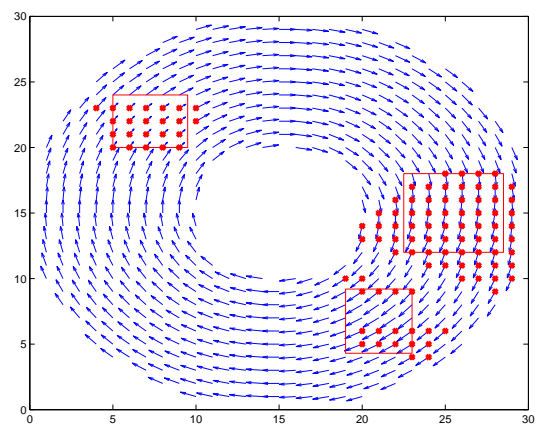

(b) Dots represent the detected abnormalities.

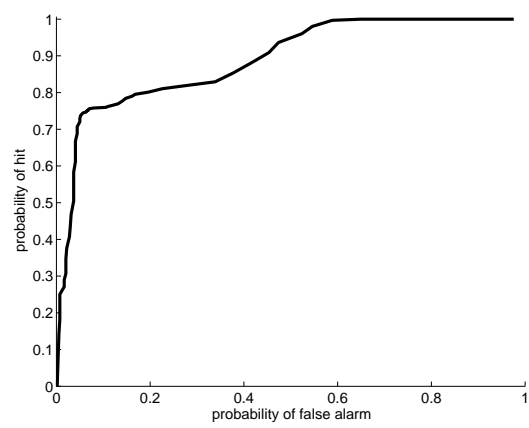

(c) ROC curve of the classifier.

Fig. 2. Experimental results using synthetic data.

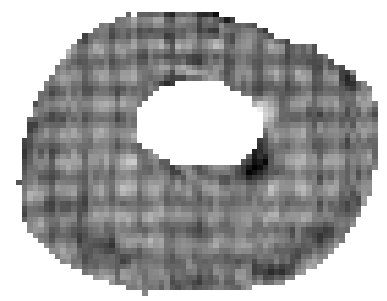

(a) Cardiac MR image.

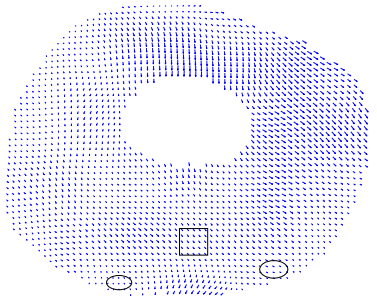

(b) Motion map

Fig. 3. A rat heart imaged on post-transplantation day 3.

marked by triangles in Fig. 4, the epicardial regions show good agreement with our detected abnormal regions. This verification shows that our approach is promising to detect early rejection of heart transplants.

\section{CONCLUSIONS}

In this paper, we develop a semi-supervised algorithm to classify early rejection of heart transplants. We use a graph based model to represent the motion data and use this model to design our algorithm. Classification is achieved by computing the eigenfunctions of the graph Laplacian, which we use to express the classifier. Human-labeled data trains the classifier. Experimental results with real cardiac MRI images demonstrate that our approach will be a helpful tool for early rejection diagnosis.

\section{REFERENCES}

[1] Y. L. Wu, K. Sato, J. B. Williams, K. Hitchens, H.H. Chang, J. M. F. Moura, and C. Ho, "The acute cardiac allograft rejection is heterogeneous and can be non-invasively monitored in vivo with MRI by the twopronged method," in Proceedings of Annual Scientific Sessions of the Society for Cardiovascular Magnetic Resonance, San Francisco, CA, January 2005.

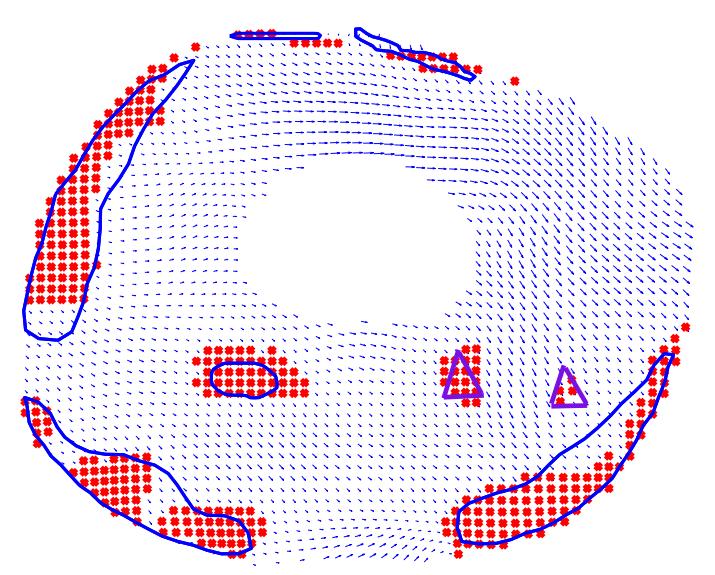

Fig. 4. Experimental results using real cardiac MRI data.

[2] F. R. K. Chung, Spectral Graph Theory, vol. 92 of CBMS Regional Conference Series in Mathematics, American Mathematical Society, 1997.

[3] M. Belkin and P. Niyogi, "Laplacian eigenmaps for dimensionality reduction and data representation," Neural Computation, vol. 15, no. 6, pp. 1373-1396, 2003.

[4] M. Belkin and P. Niyogi, "Semi-supervised learning on Riemannian manifolds," Machine Learning, vol. 56, pp. 209-239, 2004.

[5] R. O. Duda, P. E. Hart, and D. G. Stork, Pattern Classification, John Wiley \& Sons, New York, NY, second edition, 2001.

[6] H.-H. Chang, J. M. F. Moura, Y. L. Wu, K. Sato, and C. Ho, "Reconstruction of 3-D dense cardiac motion from tagged MR sequences," in Proceedings of IEEE International Symposium on Biomedical Imaging, Arlington, VA, April 2004, pp. 880-883. 